Fourth International Conference on Sustainable Construction Materials and Technologies http://www.claisse.info/Proceedings.htm

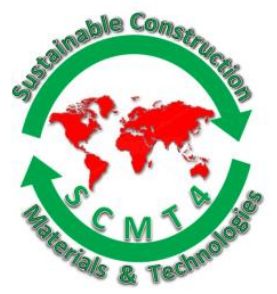

SCMT4

Las Vegas, USA, August 7-11, 2016

\title{
A Review on Microstructure Characterization of Cement-Based Materials Subjected to Chloride by AC Impedance
}

\author{
Xiang. Hu ${ }^{1 a, 2 a}$, Caijun. Shi ${ }^{1 b}$, and Geert. De Schutteror ${ }^{2 b}$ \\ ${ }^{I}$ College of Civil Engineering, Hunan University, Changsha 410082, PR China. \\ ${ }^{2}$ Magnel Laboratory for Concrete Research, Department of Structural Engineering, \\ Ghent University, Ghent B-9052, Belgium. ${ }^{1 a, 2 a}$ Email: <huxiang.gent@gmail.com>, \\ ${ }^{1 b}$ Email: 〈caijunshi@yahoo.com>, ${ }^{2 b}$ Email: <geert.deschutter@ugent.be>.
}

\begin{abstract}
AC impedance spectroscopy is a powerful technique, and has been widely applied to measure microstructure and electrochemical properties of cement-based materials. This paper reviews the equivalent circuit models proposed by researchers to study the chloride related issues, including microstructure based models and electrical circuit models corresponding to ion migration and steel corrosion. The determination of the chloride induced corrosion parameters such as chloride diffusion coefficient, chloride concentration threshold and corrosion rate, and the microstructural modification of cement-based materials due to the chloride penetration in chloride migration test are all introduced. The inhomogeneous structure of cement-based materials make it difficult to determine an appropriate equivalent circuit model. An systemic and instructive methodology for the determination of equivalent circuit for AC impedance spectroscopy analysis is needed.
\end{abstract}

\section{INTRODUCTION}

Chloride induced steel corrosion has become one of the main deterioration problem for reinforcing steel structure, especially for the structures exposed to offshore environment or where de-icing salt is used. A variety of techniques were used in determining the chloride penetration parameters and microstructural evolution of cement-based materials after chloride penetration. However, there are lots of limitations to the application of these methods results from longer testing time consumption and/or microstructural variation due to the application of electric field and reaction between chloride ions and testing samples during testing periods. Plenty of studies have been conducted to determine the modification of concrete microstructure induced by chloride. These techniques, which including MIP, SEM and X-ray diffraction (XRD), are destructive and local. It is not allowed to determine the changes at different time and reflect the overall properties of a relative large samples.

Essentially, the corrosion of reinforcing steel in concrete belongs to an electrochemical process. In 1959, half-cell potential measurement was applied to evaluate the influences of concrete admixtures on steel corrosion in the study of Kaesche [1959]. After that, electrochemical techniques including linear polarization resistance (LPR), AC impedance spectroscopy (EIS), electrochemical noise measurements (EN) and galvanostatic pulse measurements have been used in the research related to chloride induced 
corrosion and microstructural modified by chloride penetration. Among these, AC impedance spectroscopy techniques received most significant attention and was widely applied to study the chloride transportation parameters, corrosion rate and chloride concentration threshold in reinforcement concrete, and influences of chloride penetration on microstructure of cement-based materials during nature diffusion and electrical acceleration migration process.

AC impedance spectroscopy is a non-destructive method for the measurement of electrochemistry properties of materials since 1980s [McCarter 1988]. It allows real time monitoring of the same cementbased material samples at different periods and the testing is universal. The macroscopic characteristics of cement-based materials, such as bulk resistance, resistance of pore solution and capacitance of double layer in the pore solution, can be reflected on AC impedance spectrum and obtained by fitting with an appropriate equivalent circuit model. AC electrochemical impedance spectroscopy (EIS) has been demonstrated to be a promising technique for revealing the hydration of cementitious materials and formation of pore structure, and providing useful information for the evaluation of mechanical properties, permeability and durability.

This paper reviews the application of electrochemical impedance spectroscopy in determining the chloride migration parameters and microstructural evolution of cement-based materials after chloride penetration and the proposed equivalent circuit models for chloride related studies. The determination of chloride diffusion coefficient by using different EIS parameters is summarized.

\section{EQUVALENT CIRCUIT MODELS FOR CHLORIDE ION MIGRATION}

Complicated electrochemical systems form in cement-based materials when just a small electrical voltage is applied, of which can be expressed by parallel or series connection of resistor, capacitor and/or inductor. Equivalent circuit is the most important tool for correlate the electrical response and microstructure of cement-based materials. In a typical Nyquist plot of cement-based materials, several capacitive loop can be found, and every loop is simulated with a parallel series of resistance and capacitance. However, the inhomogeneous inner structure of concrete makes it difficult to find an appropriate equivalenr circuit model. Several equivalent circuit models have been proposed by researchers, but few of them are totally convincing in interpreting the experimental results. After being penetrated by chloride ions, new products will produced within cement paste, mortar or concrete. The microstructure and properties of interface between solids and liquid may also be changed to some extent, which may affect the value of electrical circuit parameters or even the corresponding equivalent circuit.

Actually, among the proposed models, most of them are based on the microstructure of materials, even for some models related to chloride penetrated process. B. Diaz et al [2006] studied the impedance spectrum of mortar samples using two and four electrode arrangement in a permeation cell (Figure 1) and corresponding equivalent circuit model showed in Figure 2. For four electrode measurement, the internal reference electrode located close to the surface of sample and lead to the remove of parallel series of $\mathrm{C}_{3}$ and $\mathrm{R}_{3}$ in equivalent circuit. Two electrode impedance measurement were widely used in AC impedance researches on microstructure and chloride-induced corrosion of cement-based materials due to the larger frequency window. The total impedance expression $Z(\omega)$ of the model employed for two electrode measurement can be:

$Z(\omega)=R_{e}+\frac{Z_{1} \cdot Z_{2}}{Z_{1}+Z_{2}}+\frac{R_{3}}{1+\left(j \omega R_{3} C_{3}\right)^{\alpha 3}}$ where $Z_{1}=\frac{R_{1}}{1+\left(j \omega R_{1} C_{1}\right)^{\alpha 1}}$ and $Z_{2}=R_{2}\left(1+\left(j \omega R_{2} C_{2}\right)^{-\alpha 2}\right)$

$R_{e}$ is the resistance of electrolytes between the sample and the electrode; $R_{1}$ is resistance attributed to the ionic motion in pores that connected both end faces of the sample; $R_{2}$ corresponds to the resistance attributed to the ionic motion in unconnected pores; $\mathrm{C}_{1}$ and $\mathrm{C}_{2}$ are related to the solid fraction and double 
layer effects at pore walls; $\mathrm{R}_{3}$ and $\mathrm{C}_{3}$ represent the resistance and capacitance associated with the electrolyte-concrete interface. $Z_{1}$ and $Z_{2}$ is impedance of the parallel and series branch, relatively.

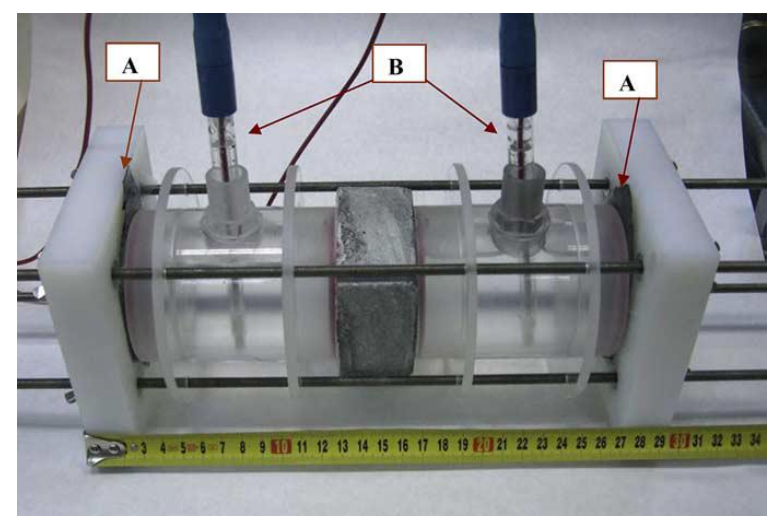

Figure 1. The permeation cell employed impedance measurement.

(A)External graphite electrode for applying the electric field. (B) Inner reference electrodes for four electrode impedance measurements.

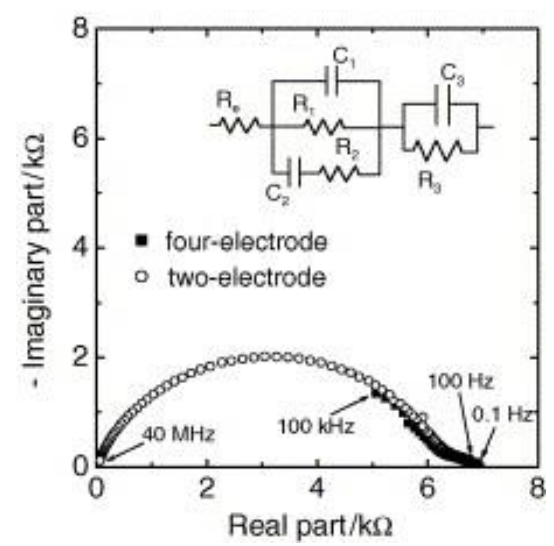

Figure 2. Impedance measurements on a $4 \mathrm{~cm}$ thick mortar sample using two and four electrode arrangement. The measurements were performed after $7 \mathrm{~h}$ migration test $(5 \mathrm{~V}$ DC), using $0.5 \mathrm{M} \mathrm{NaCl}$ electrolyte. The equivalent circuit employed to model two-electrode data is also depicted.

Cause the chloride migration properties of samples have close relationship with the microstructure, it can be directly represented by parallel or series connection of different electrical elements. The value of resistance or capacitance in equivalent models are used to calculate the chloride diffusion coefficient, corrosion rate or other chloride related parameters, which will review in next part. In 2012, Hugo Mercado et al [2012] simplified the microstructural based model into the one depicted in Figure 3 which was made of the association of the electrical properties of electrode(E) and testing materials(mat). In their experiment, electrode and testing sample was directly contacted with each other without electrolyte. The frequency dependent impedance $(Z(\omega))$ of this equivalent circuit is given by the following expression:

$Z(\omega)=Z_{\text {real }}(\omega)+i Z_{\text {im }}(\omega)$ where $Z_{\text {real }}(\omega)=\frac{2 R_{E}}{1+\left(\omega R_{E} C_{E}\right)^{2}}+\frac{R_{\text {mat }}}{1+\left(\omega R_{\text {mat }} C_{\text {mat }}\right)^{2}}$ and $Z_{\text {im }}(\omega)=$ $\frac{2 \omega R_{E}^{2} C_{E}}{1+\left(\omega R_{E} C_{E}\right)^{2}}+\frac{\omega R_{\text {mat }}^{2} C_{\text {mat }}}{1+\left(\omega R_{\text {mat }} C_{\text {mat }}\right)^{2}}$

Where, $Z_{\text {real }}$ and $Z_{\text {im }}$ is the real and imaginary component of the impedance. 


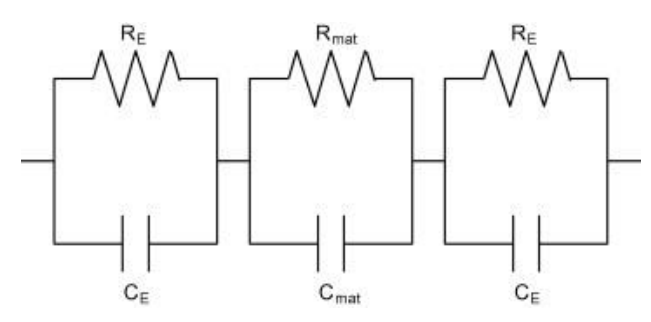

Figure 3. A simplified equivalent circuit model

Generally, two capacitive loops are include in the Nyquist plot related to equation (2). With a typical frequency when the value of $Z_{\text {im }}$ equals to 0 , the impedance of the system can be transferred to:

$$
Z(\omega)=Z_{\text {real }}(\omega)=R_{\text {mat }}
$$

Practically, the value of imaginary part is not null under the condition of equation (3) due to the non-ideal behavior of the system. We consider the real part of the point related to minimal value of $Z_{\mathrm{im}}$ as $R_{\mathrm{mat}}$, which is also known as bulk resistance of the materials.

For some complicated systems, replace the pure capacitance element by constant phase angel element(CPE) may to some extent eliminate the dispersion effect due to the roughness of the surface of solids or electrode. A CPE equals to a distributing parameter circuit, and can be treated as an imperfect capacitor. In Nuquist plot, the parallel connection of resistant and CPE is showed as an arc whose center point deviated from the $\mathrm{x}$ axis. The impedance of CPE can be expressed by:

$$
Z=\frac{1}{Y_{0}} \omega^{-n}\left(\cos \frac{n \pi}{2}-j \sin \frac{2 \pi}{2}\right) \quad(0<\mathrm{n}<1)
$$

Where, $\mathrm{Y}_{0}$ and $n$ are parameters of CPE. The value of $n$ is related to the deviation of CPE from pure capacitance. When $n=1$, the system is described by a single time-constant and the parameter $Q$ has units of capacitance; otherwise, $Q$ has units of $\mathrm{s}^{\mathrm{n}} / \Omega \mathrm{cm}^{2}$ or $\mathrm{Fs}^{\mathrm{n}-1} / \mathrm{cm}^{2}$ [Orazem 2011]. In the research of Olivier et al [2004], an equivalent circuit model (Figure 4) including CPE was used to determine the chloride concentration threshold. However, the meanings of other circuit parameters except polarization resistance were not gave out.

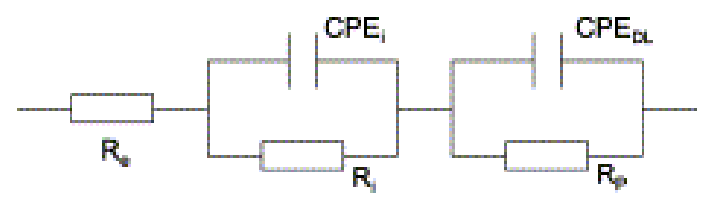

Figure 4. Equivalent circuit model for impedance simulation

The penetrated chloride within cement-based materials will change the properties of pore solution and solid-liquid interface. Meanwhile, the reaction of ions with solid phase may also affect the inner pore structure of materials. Therefore, equivalent circuit models based on the ion migration within cementbased materials were also proposed, especially for studies related to steel corrosion process. As shown in Figure $5, R_{\mathrm{s}}$ is the resistance of electrolyte, $C_{\mathrm{d}}$ accounts to capacitance of the electrode/electrolyte interface. Faradaic impedance $Z_{\mathrm{F}}$ (as shown in oblong box) here was used to characterize the reaction kinetics and diffusion, which is composed of the resistance of charge transfer $R_{\mathrm{ct}}$ in series with the socalled Warburg impedance $Z_{\mathrm{W}}$ describing the diffusion behavior. The total impedance of circuit model showed in figure can be expressed as follow:

$$
Z=R_{S}+\frac{Z_{F}}{1+i \omega Z_{F} C_{D}} \text { where } Z_{F}=R_{c t}+Z_{W} \text { and } Z_{W}=\sigma \omega^{-\frac{1}{2}}(1-i)
$$




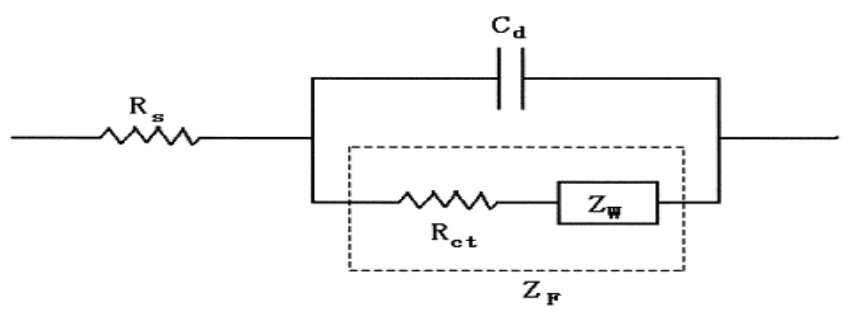

Figure 5. Equivalent circuit based on ion migration

An appropriate equivalent circuit model is very important to AC impedance spectroscopy analysis. The application of circuit model in AC impedance spectrum analysis makes it more feasible and convenient to monitor the microstructural and electrochemical properties changes during and after chloride migration. However, the inhomogeneous and complex component and structure of cement-based materials result into the indeterminacy of equivalent circuit model for a typical system. For one set data, several models may be fitted. The hydration of cement do have significant influences on performance of samples, which may sometimes change the corresponding best fitted model. In some studies, different circuits were provide and data fitting were all done for these models to find the most suitable one for different samples. However, the absence of an systemic and instructive methodology for the determination of equivalent electric circuit model for AC impedance spectroscopy analysis restricts the application of AC impedance technique.

\section{CHLORIDE INDUCE CORROSION PARAMETERS DETERMINATION BASED ON AC IMPEDANCE}

When exposed to the chloride environment, the reinforced steel concrete structure may simultaneously experience plenty of actions of chemical, physical and electrochemical. However, the corrosion process of steel is mainly related to electrochemical action. The results of AC impedance spectroscopy may reveal information about the reaction mechanism of an electrochemical process: different reaction steps will dominate at certain frequencies, and the frequency response can help identify the rate limiting step.

The Warburg impedance element is a common diffusion circuit element that can be used to model semiinfinite linear diffusion, that is, unrestricted diffusion to a large planar electrode. The relation between chloride diffusion coefficient $D$ and coefficient of Warburg impedance $\sigma$ is:

$$
\begin{gathered}
\sigma=\frac{R T}{F^{2} A \sqrt{2} D^{1 / 2} C} \\
\text { Hence, } D=\left(\frac{R T}{\sqrt{2} \sigma F^{2} A C}\right)^{2}
\end{gathered}
$$

Where $R$ is the gas constant, $T$ is the absolute temperature, $F$ is Faraday's constant, $A$ is the area of electrode surface, and $C$ is concentrations of chloride. By using the AC impedance properties in the lowfrequency region in spectrum, Shi et al [1999] studied the chloride diffusivity in concrete with different strength grade and the change of chloride diffusion coefficient with time. However, this method is not able to distinguish the migration of chloride ions from other species of ion. The results obtained in this method maybe the diffusion coefficient of all the aggressive ions.

Based on the Einstein-Smoluchowski equation and the assumption that the contribution to conductivity of the intrinsic ions in pore solution can be neglected, Diaz et al [2006] proposed a method to calculated the chloride diffusion coefficient from the resistance of percolating pores $R 1$.

$$
u_{j}=\frac{z_{j} F}{R T} D_{j} \text { being } \rho=\frac{R_{1}}{a} \text { and } \frac{1}{\rho}=F \sum_{j} z_{j} u_{j} C_{j}
$$




$$
\text { Hence, } D_{C l-}=\frac{R T a}{\left(1+\frac{u_{N a+}}{u_{C l-}}\right) F^{2} C_{N a C l} * R 1}
$$

Where $z_{\mathrm{j}}$ relates to the charge of ion $j\left(\mathrm{Na}^{+}\right.$and $\left.\mathrm{Cl}^{-}\right), C_{\mathrm{j}}$ is ion concentration (assuming activity coefficient close to 1$), u_{\mathrm{j}}$ is the ionic mobility, $a$ is sample's cell constant (thickness to area ratio). The value of $u_{\mathrm{Na}+} / u_{\mathrm{Cl}}$ can be obtained in handbooks of chemistry for idea solutions, or through measuring the liquid junction potential of solutions.

Recently, Hugo et al [2012] proposed a method to calculate the diffusion coefficient of an ionic species from the saturated material resistance. The Nernst-Einstein relation and formation factor was used in this method. The ratio of the pore solution conductivity $\sigma_{\text {pore }}$ to the conductivity of the material saturated with the same pore solution $\sigma_{\text {mat }}$ equals to the ratio of ion diffusion coefficient in the pore solution $D_{\text {pore }}$ to that of the same ion through the saturated material, D. This ratio was known as the formation factor F. This approach which would be questionable for other materials was proven to be valid for cement-based materials by Refs. [Snyder et al 2000 and Snyder 2001].

$$
F=\frac{\sigma_{\text {pore }}}{\sigma_{\text {mat }}}=\frac{D_{\text {pore }}}{D}
$$

In their study, pore solution extractions was performed to measure the ionic composition of pore solution. After that, an artificial solution was prepared according to the results of ionic composition analysis of expressed pore solution. Then, the conductivity and chloride diffusion coefficient of this artificial solution was determined. The conductivity of materials can be determined by results of AC impedance spectroscopy from:

$$
\sigma_{\text {mat }}=\frac{L}{A R_{\text {mat }}}
$$

Where $L$ is the sample thickness $(\mathrm{m}), A$ is the sample cross-section $\left(\mathrm{m}^{2}\right)$, and $R_{\text {mat }}$ is the saturated material resistance $(\Omega)$ measured from the EIS experiments. The chloride diffusion coefficient measured by this method was compared with another two technical, migration test and current measurement, AC impedance spectroscopy measurement provides comparable values with those obtained by traditional diffusion or migration techniques.

The high alkalinity of the interstitial solution of concrete can protect the reinforcing steel from corrosion. Chloride concentration threshold is the concentration of chloride ions near the rebar which may initiate the steel corrosion. Poupard et al[2004] measured the low-frequency impedance response of concrete samples at various curing time, they found that the sharply decrease of the capacitive part was related to the corrosion initiation, which was demonstrated by the results of SEM observations and multielementary analyses. The chloride profile within concrete was calculated according to Tang and Nilsson [1993] and $\mathrm{Xu}$ and Chandre [1994]. For the initial condition $\mathrm{C}(\mathrm{x}, \mathrm{t}=0)=0$ and boundary condition $C(\mathrm{x}=0, \mathrm{t})=\mathrm{C}_{0}$ (constant value), chloride distribution at a depth $x$ and a time $t$ can be expressed by:

$$
\begin{gathered}
\frac{C(x, t)}{C_{0}}=\frac{1}{2} e^{\frac{z_{C l}-F \mathrm{E}_{e}}{2 R T} X}\left[e^{-\beta x} \operatorname{erfc}\left(\frac{x-2 \beta D_{n s s} t}{2 \sqrt{D_{n s s} t}}\right)+e^{-\beta x} \operatorname{erfc}\left(\frac{x+2 \beta D_{n s s} t}{2 \sqrt{D_{n s s} t}}\right)\right] \\
\text { Being } \beta=\sqrt{\left(\frac{z_{C l-F}}{2 R T} E_{e}\right)^{2}+\frac{k}{D_{n s s}}}
\end{gathered}
$$

Where $C_{0}$ is the chloride concentration in the solution in contact with the cement-based material $\left(\mathrm{mol} / \mathrm{m}^{3}\right)$, $x$ is the depth $(\mathrm{m}), E_{\mathrm{e}}$ is the external electrical field, $D_{\mathrm{nss}}$ is the non-steady-state diffusion coefficient. $k$ is the binding rate between chlorides and hydrated components of the cement-based material (mainly $\mathrm{C}_{3} \mathrm{~A}$ ). As referred to in reference [Tang and Nilsson 1996], $D_{\mathrm{nss}}=D_{\mathrm{ss}} /\left[\varepsilon_{\mathrm{p}}(1+B)\right]$, where $\varepsilon_{\mathrm{p}}$ is the porosity of the 
cement-based material and $B$ is called chloride-binding capacity. As shown in their study, the beginning of $R_{\mathrm{p}}$ drop was proved occurred simultaneously with the propagation period (the ignition of steel corrosion). The chloride concentration required to active the corrosion and the time when steel corrosion started can be determined according to the results of equation with the time of $R_{\mathrm{p}}$ start to decrease and the depth of concrete cover. The chloride concentration threshold of the testing concrete samples was increased as the ratio of water/cement decreasing.

Generally, it's necessary to detect the corrosion at early stages to control and maintain the quality of concrete structure and arrange the repairing work. Rapid and accurate methods for measuring corrosion rates are of tremendous importance, especially for practical applications. Many researches have been proposed to evaluate the corrosion rate of a metal based on electrochemical method and Faraday's low. The relationship of corrosion rate $(C R)$ of steel and the density of corrosion current $i_{\text {corr }}$ can be derived from the Faraday's low,

$$
C R(\mu m / y)=\frac{3.27 \times i_{c o r r} \times E . W}{d}
$$

Where E.W. represents the equivalent weight of steel (gm) and $d$ is the density of reinforcing bar $\left(\mathrm{gm} / \mathrm{cm}^{3}\right)$. Nowadays, the study of corrosion rate of reinforcing steel embedded in concrete was conducted by using AC impedance spectroscopy via Stern-Geary equation:

$$
i_{\text {corr }}=B / R_{p} \text { being } B=\frac{b_{a} b_{c}}{2.303\left(b_{a}+b_{c}\right)}
$$

Where $B$ is the Stern-Geary constant, $b_{\mathrm{a}}$ and $b_{\mathrm{c}}$ are the Tafel slopes for the anodic and cathodic reactions, respectively. The resistance related to the steel-solid surface and the corrosion products $R_{\mathrm{p}}$ can be obtained from the results of AC impedance measurements. A laboratory study [Mohamed and Masayasu 2006] was conducted to determine the corrosion rate of reinforcing steel embedded in ordinary Portland concrete and high performance concrete and the results were compared with some other techniques, such as Tafel plot and linear Polarization resistance (LPR). The results demonstrated a reasonable agreement among the results of AC impedance, TP and LPR. Meanwhile, lower corrosion rate was tested for HPC specimens comparing to that of OPC, and drying cycles was confirmed be able to accelerate corrosion rate significantly. The same work was also done by Pradhan and Bhattachariee [2009] by using AC impedance, LPR and gravimetric measurements and the results were confirmed by analysis of variance (ANOVA). The variations of $i_{\text {corr }}$ measured by these measurements can be expressed as:

$$
i_{\text {corr }}(L P R)=1.10 \times i_{\text {corr }}(\text { AC impedance })=0.99 \times i_{\text {corr }}(\text { gravimetric })
$$

Due to the limits of testing equipment, studies based on AC impedance measurements were not so popular, and sometimes Nyquist diagram may only have a constant phase angle line without an arc at the high-frequency side. However, with the development of electrochemical measurements, AC impedance spectroscopy measurement has become a powerful technique to study the electrochemical properties of steel/concrete system. Cooperate with an appropriate equivalent circuit model, AC impedance plot provides useful information about ion migration, interfacial/pitting corrosion. The application of AC impedance technique is of fundamental interest for detail knowledge of steel corrosion studies.

\section{MICROSTRUCTURAL MODIFICATION DUE TO CHLORIDE MIGRATION FOLLOWED BY AC IMPEDANCE}

As we can see form the first part of this paper, parameters in equivalent circuit models are in close relationship with the microstructure of cement-based materials. The penetrated chloride ions change the 
composition and concentration of pore solution, which may affect the resistance of pore solution. Meanwhile, chloride ions in pore solution may be physically absorbed onto the surface of hydration products or react with solids (mainly aluminates) and produced Freidel's salt, which relates to the value of capacitance of the interface and bulk resistance of cement-based materials.

Studies on microstructure of cement-based materials by AC impedance spectroscopy are mainly based on the relation between the effective conductivity $\sigma_{\text {eff }}$ of samples and the bulk resistance $R_{\mathrm{b}}$ obtained from $\mathrm{AC}$ impedance spectroscopy (abscissa of the intersection of the bulk and electrode arcs in a Nyquist plot) as:

$$
\sigma_{e f f}=\frac{L}{R_{b} A}
$$

Where $L$ and $A$ are the length and effective testing area of samples respectively.

The obtained effective conductivity can be used to evaluate the diffusion properties of samples, and also has close relation with pore structure as:

$$
\sigma_{e f f}=\sigma_{\text {pore }}(\phi \beta)
$$

Where $(\phi \beta)$ is lumped pore structure parameter, which is the product of porosity $(\phi)$ and pore connector factor $(\beta)$, opore is pore solution conductivity.

In the study of Neithalath and Jain [2010], two conductivity ratios are defined:

$$
\begin{gathered}
\sigma_{\text {eff }}^{*}=\frac{\left(\sigma_{\text {eff }}\right)_{\text {modified-concrete }}}{\left(\sigma_{\text {eff }}\right)_{\text {plain-concrete }}} \\
\sigma_{\text {pore }}^{*}=\frac{\left(\sigma_{\text {pore }}\right)_{\text {modified-concrete }}}{\left(\sigma_{\text {pore }}\right)_{\text {plain-concrete }}}
\end{gathered}
$$

In these two equations, modified-concrete means silica fume or fly ash modified concrete mixtures while plain-concrete relates to plain concrete mixture without admixtures. Dividing Eq. (18b) by Eq. (18a) and substituting Eq. (17) gives:

$$
\frac{\sigma_{\text {pore }}^{*}}{\sigma_{\text {eff }}^{*}}=\frac{(\phi \beta)_{\text {plain-concrete }}}{(\phi \beta)_{\text {modified-concrete }}}=(\phi \beta)^{*}
$$

The value of $(\phi \beta)^{*}$ can be used to evaluate the pore structure refinement due to the replacement of admixtures. The results showed higher $(\phi \beta)^{*}$ value than 1.0 for samples with fly ash at later ages and silica fume at all ages, indicating pore structure refinement for modified concrete. However, the microstructural changes for fly ash modified concrete did not occur at early ages due to the low pozzolanic reaction of fly ash. With the addition of vitreous calcium alumino-silicate (VCAS), another study [Jain and Neithalath 2011] was also conducted to follow the microstructural parameter $(\phi \beta)$ change after the replacement of admixtures. As shown in Figure 6, the values of $\phi \beta$ is gradually decreased with the curing duration for all 4 groups. Except the samples with fly ash at early ages, the lower values of $\phi \beta$ as compared to plain concrete was showed. Besides, microstructure of plain and fly ash modified concretes, and that of silica fume and VCAS modified concretes belonged to two separate groups, which may due to the moderately pozzolanic reactivity of fly ash and highly activity of VCAS and silica fume.

Idealizing all the connected pore of a sample into one single pore with entire features retained [Neithalath and Jain 2010] and applying the equation. (16) into the system of the idealized single pore as:

$$
R_{c}=\frac{L_{c o n n}}{\sigma_{\text {conn } A_{c o n n}}}
$$

The value of connected pore conductivity $\sigma_{\text {conn }}$ can be treated equal to pore solution conductivity $\sigma_{\text {pore }}$. However, the geometry of porosity will also affects the conductivity, and the actual value of $\sigma_{\text {conn }}$ may be 
lower than $\sigma_{\text {pore. }} L_{\text {conn }}$ is the idealized connected pore length, which $L_{\text {conn }}=L \tau$, where $\tau$ is the tortuosity of the idealized connected pore. The value of $\tau$ can be calculated as follow:

$$
\tau^{2}=\frac{\sigma_{\text {pore }}}{\sigma_{\text {eff }}} \phi
$$

Substituting equation (17) and (21) into equation (20), a simplified equivalent connected pore diameter $\left(d_{\text {conn }}\right)$ is provided as:

$$
d_{\text {conn }}=2 \sqrt{\frac{L \beta^{-1 / 2}}{\pi R_{c} \sigma_{\text {pore }}}}
$$

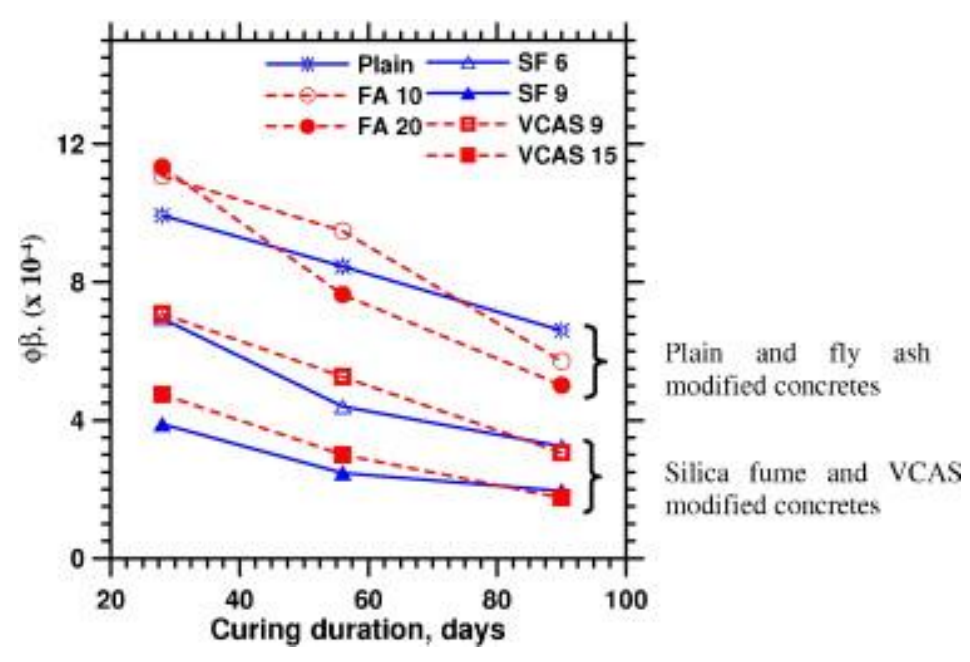

\section{Figure 6. Evolution of microstructural parameter for different mixtures at different curing duration}

The electric fields has been widely used to accelerate the chloride migration in rapid chloride migration test and determine the chloride penetration resistance of concrete. However, the applied electric fields may introduce some microstructural variations to concrete. In the last part, AC impedance technique was introduced to replace forced migration test to evaluate the chloride migration parameters of cement-based materials. Moreover, the modifications of microstructure during the electric accelerated migration test can be evaluated and quantified by testing results of AC impedance. Sanchez et al [Sánchez et al 2008] performed a migration experiments based on the set-up and procedure described in reference [Castellote 2001] and followed the microstructural changes during migration text according to the evolution of electric impedance parameters in equivalent circuit model. The evolutions of the dielectric parameters showed that during the migration test, the concrete sample experienced a saturation process with chloride solution during the initial part, then new solid phases produced which reduced the pore diameter and narrowed the pore structure. During the non-stable state migration (NSSM) test, different applied initial potential will need to applied due to the differences in microstructure of samples, which may bring different effects on microstructural modification during test. Jain and Neithalath [2011] proposed an equation to convert the bulk resistance of samples after NNSM test with different applied potential $\left(\mathrm{R}_{\mathrm{b}-}\right.$ v) after NSSM to that with a typical applied potential (such as $30 \mathrm{~V}$, which equals to $600 \mathrm{~V} \mathrm{~m}^{-1}$ in their study) $\left(\mathrm{R}_{\mathrm{b}-30 \mathrm{v}}\right)_{\text {after NSSM. }}$.

$$
\left(R_{b-30 V}\right)_{a f t e r ~ N S S M}=\left(R_{b-V}\right)_{a f t e r ~ N S S M}\left[1+\frac{\Delta i_{c a l-30 V}}{i_{n e w-30 V}}\right]
$$


Where inew-30V is the current of samples after NSSM test with a new voltage (30V), $\Delta i_{\text {cal-30V }}$ is the corrected difference in current due to the different applied potential (x and 30V), which can be calculated by multiplying $\Delta i_{\text {exp }}$ to ratio of the penetration depth [(xd)cal-30V/(xd)exp].

\section{CONCLUSION}

Impedance spectroscopy measurements on cement-based materials are of great significance due to the possibility of establish the relationship between the circuit parameters and the microstructure of the system. The application of AC impedance spectroscopy techniques have received extensive concern in the field of microstructure assessment and steel corrosion issues. The chloride ion will affect the microstructure and electrochemical properties of cement-based system and the AC impedance techniques has been applied in chloride induced corrosion for a long period. This paper reviewed the determination of equivalent circuit models, which is important for the analysis of impedance plot and the widespread application of AC impedance spectroscopy techniques. The methods to calculate chloride migration and corrosion parameters are introduced.

However, the existence studies are still failed to find a convincing method for electric circuit model determination. The complicated structure of cement-based materials makes it difficult to model the system with a certain equivalent circuit model. Further studies are of significantly needed for the determination of equivalent circuit models. On one hand, the number of time constants in impedance spectrum should be identified and the circuit model should be proposed with a priority consideration of the microstructure and the potential conductive paths in cement-based materials to guarantee a practical physical meaning to the circuit parameter. On the other hand, a systematic research on performance of cement paste, mortar and concrete at a longer hydration time with a wide range of frequency are required.

The experimental condition and testing facilities are very important and significantly affect the testing results of AC impedance spectroscopy measurements. However, the research on these fields are insufficient in previous studies. Systematic studies on experimental condition control and testing method determination are strongly needed.

\section{REFERENCES}

Kaesche, H. (1959). "Standard test method for half-cell potentials of uncoated reinforcing steel in concrete." Zem.-Kalk-Gips, 12, 289.

McCarter, W. J., Garvin, S. and Bouzid, N. (1988). “Impedance measurements on cement paste.” Journal of materials science letters, 7(10), 1056-1057.

Díaz B, Nóvoa X R, Pérez M C. (2006). "Study of the chloride diffusion in mortar: a new method of determining diffusion coefficients based on impedance measurements." Cement and Concrete Composites, 28(3), 237-245.

Mercado, H., Lorente, S. and Bourbon, X. (2012). "Chloride diffusion coefficient: A comparison between impedance spectroscopy and electrokinetic tests." Cement and Concrete Composites, 34(1), 68-75.

Orazem, M. E. and Tribollet, B. (2011). "Electrochemical impedance spectroscopy." Vol. 48, John Wiley $\&$ Sons.

Poupard, O., Ait-Mokhtar, A. and Dumargue, P. (2004). "Corrosion by chlorides in reinforced concrete: Determination of chloride concentration threshold by impedance spectroscopy." Cement and Concrete Research, 34(6), 991-1000. 
Shi, M., Chen, Z. and Sun, J. (1999). "Determination of chloride diffusivity in concrete by AC impedance spectroscopy." Cement and Concrete Research, 29(7), 1111-1115.

Díaz, B., Nóvoa, X. R. and Pérez, M. C. (2006) "Study of the chloride diffusion in mortar: a new method of determining diffusion coefficients based on impedance measurements." Cement and Concrete Composites, 28(3), 237-245.

Neithalath, N. and Jain, J. (2010). "Relating rapid chloride transport parameters of concretes to microstructural features extracted from electrical impedance." Cement and Concrete Research, 40(7), 1041-1051.

Jain, J. and Neithalath, N. (2011). "Electrical impedance analysis based quantification of microstructural changes in concretes due to non-steady state chloride migration." Materials Chemistry and Physics, 129(1), 569-579.

Snyder, K. A., Ferraris, C., Martys, N. S., et al. (2000). "Using impedance spectroscopy to assess the viability of the rapid chloride test for determining concrete conductivity." JOURNAL OF RESEARCH-NATIONAL INSTITUTE OF STANDARDS AND TECHNOLOGY, 105(4), 497-510.

Snyder, K. A. (2001). "The relationship between the formation factor and the diffusion coefficient of porous materials saturated with concentrated electrolytes: theoretical and experimental considerations." Concrete Science and Engineering, 3(12), 216-224.

Pradhan, B. and Bhattacharjee, B. (2009). "Performance evaluation of rebar in chloride contaminated concrete by corrosion rate." Construction and building materials, 23(6), 2346-2356.

Ismail, M. and Ohtsu, M. (2006). "Corrosion rate of ordinary and high-performance concrete subjected to chloride attack by AC impedance spectroscopy." Construction and Building Materials, 20(7), 458469.

Sánchez, I., Nóvoa, X. R., de Vera, G., et al. (2008) "Microstructural modifications in Portland cement concrete due to forced ionic migration tests. Study by impedance spectroscopy." Cement and concrete research, 38(7), 1015-1025.

Castellote, M., Andrade, C. and Alonso, C. (2001). "Measurement of the steady and non-steady-state chloride diffusion coefficients in a migration test by means of monitoring the conductivity in the anolyte chamber. Comparison with natural diffusion tests." Cement and Concrete Research, 31(10), $1411-1420$.

Tang, L., Nilsson, L.(1993). "Rapid determination of the chloride diffusivity in concrete by applying an electric field.” ACI materials journal, 89(1).

$\mathrm{Xu}, \mathrm{A}$., Chandra, S. "A discussion of the paper "calculation of chloride diffusion coefficients in concrete from ionic migration measurements" by C. Andrade." Cement and Concrete Research, 24(2), 375-379. 